\section{REVIEW ARTICLE}

A.J. Degnan

L.M. Levy

\title{
Pseudotumor Cerebri: Brief Review of Clinical Syndrome and Imaging Findings
}

SUMMARY: PTC is a clinical entity of uncertain etiology characterized by intracranial hypertension. The syndrome classically manifests with headaches and visual changes in women with obesity. Traditionally, imaging ruled out secondary causes of elevated CSF pressure but now may reveal findings frequently seen in patients with PTC, including the following: flattening of the globe, an empty sella, an enlarged ONS, protrusion and enhancement of the optic nerve head, and increased tortuosity of the optic nerve. Novel imaging methods, including MR venography, have additionally identified sinovenous stenosis as a potential indicator of PTC.

ABBREVIATIONS: BMI = body-mass index; $\mathrm{CN}=$ cranial nerve; HIV = human immunodeficiency virus; ICP = intracranial pressure; $I I H=$ idiopathic intracranial hypertension; ISF = interstitial fluid; MRI = MR imaging; ONS = optic nerve sheath; ONSF = optic nerve sheath fenestration; IOP = intraocular pressure; PCOS = polycystic ovary syndrome; PTC = pseudotumor cerebri syndrome

I ntracranial hypertension is a clinical entity with a myriad of known and putative etiologies. In the history of this condition, the name given to the clinical syndrome referred to as PTC or more commonly IIH has varied widely and been the subject of much contention. ${ }^{1}$ Heinrich Quincke, an early pioneer in the use of lumbar puncture, reported the first recorded cases of intracranial hypertension of unknown cause in what he described as "meningitis serosa" in 1893; at that time, he posited that inadequate CSF resorption was responsible for the syndrome, a theory that is still entertained by some researchers. ${ }^{2}$ The term "PTC" was coined in 1904 by Nonne to describe a condition characterized by symptoms associated with intracranial tumors with an unusual course of remission and subsequently termed "benign intracranial hypertension" by Foley in 1955 . $^{1}$

The absence of a clear identifiable etiology for a clinical syndrome characterized by elevated ICP exists in nearly $90 \%$ of cases, and this ambiguity inevitably has led to the replacement of the misnomer "benign" intracranial hypertension with IIH in light of the incidence of vision loss resulting from this condition. ${ }^{3}$ Despite numerous revisions during the past century, these definitions remain inadequate and limited in that some cases of IIH have identifiable etiology, such as dural venous stenosis, which has been implicated in 14\%-90\% of patients with IIH. ${ }^{4-6}$ For this reason, certain authors proposed the use of PTC as a catchment for both categories: purely IIH and that due to secondary causes of intracranial hypertension such as venous stenosis. ${ }^{5}$ We prefer the more encompassing term "PTC" to reflect diagnostic limitations in elucidating potential secondary causes of IIH, such as those listed in Table 1. ${ }^{7,8}$ Another argument proposes a classification of isolated intracranial hypertension as being either idiopathic or secondary. ${ }^{5}$

From the Department of Radiology, George Washington University Hospital, Washington, DC.

Please address correspondence to Lucien M. Levy, Department of Radiology, George Washington University Hospital, 900 23rd Street N.W., Washington, DC 20037; e-mail: llevy@mfa.gwu.edu

Indicates open access to non-subscribers at www.ajnr.org

http://dx.doi.org/10.3174/ajnr.A2404
Several formalized criteria for IIH exist in the literature and are subject to extensive debate. The Modified Dandy Criteria first incorporated the use of CT in the diagnosis of IIH, primarily as a means of excluding occult causes of intracranial hypertension previously missed in the era before diagnostic imaging. ${ }^{9}$ Friedman and Jacobson ${ }^{7}$ updated these criteria (Table 2) to reflect the advances of MR imaging and the characterization of other etiologies of intracranial hypertension such as venous thrombosis.

Additional caveats have been proposed as conditions in the diagnosis of IIH: excluding patients who have intracranial sinus venous thrombosis, a systemic condition associated with elevated right heart pressure, or those who have been exposed to medications or toxins associated with increased intracranial hypertension such as those mentioned in Table 1.,5

\section{Etiology}

The precise etiology of IIH, as the name suggests, is largely unknown, despite much clinical investigation and basic science research. Theories generally fall under 5 different proposed mechanisms resulting in elevated intracranial hypertension as outlined in Table 3 and discussed at greater length in the article by Walker. ${ }^{10}$ Older theories, including those of Quincke, suggest a disturbance of CSF homeostasis, either impairment of reabsorption or excess production of CSF. ${ }^{1,11}$ Many researchers cite case studies showing CSF collections as proof of this theory of disordered CSF hydrodynamics. ${ }^{12}$ Critics of this traditional hypothesis point to the intermittent success of lumbar puncture, which, from a physiologic standpoint, should alleviate the symptoms due to excess CSF and, in addition, the lack of expansion of ventricles, which would occur if CSF volume was increased as in the case of meningitisinduced hydrocephalus. ${ }^{10}$

Many cases of PTC may not, in fact, be idiopathic but rather secondary to venous thrombosis, which many argue is frequently missed if MR venography is not used to evaluate patients with suspected PTC. ${ }^{13}$ As a result, 1 author proposed classifying benign intracranial hypertension as either idiopathic (also called PTC) or venogenic, if caused by thrombosis, vasculitis, extrinsic venous compression, or intraluminal tumor. 8 


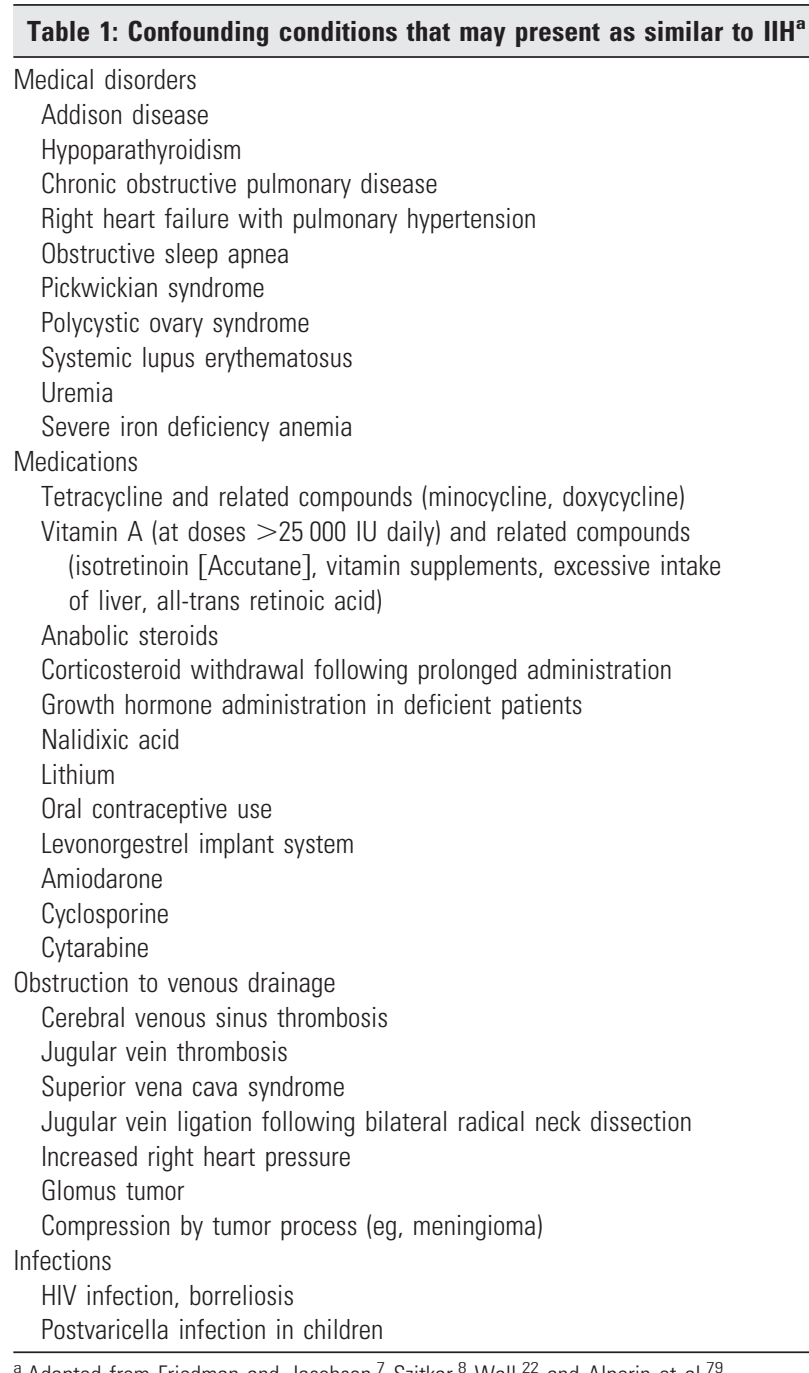

${ }^{a}$ Adapted from Friedman and Jacobson, ${ }^{7}$ Szitkar, ${ }^{8}$ Wall, ${ }^{22}$ and Alperin et al. ${ }^{79}$

A newer theory stemming from observations of sinus thrombosis producing a clinical presentation similar to IIH suggests that most cases of $\mathrm{IIH}$ are due to venous outflow obstruction, which can also take the form of stenosis in the absence of thrombosis. ${ }^{14}$ The observation of stenotic transverse sinuses in $>90 \%$ of patients with IIH in 1 study gives credence to this thinking. ${ }^{6}$ There is clinical evidence to support the idea that impaired venous outflow leads to increased total blood flow. ${ }^{6}$ Even if it is assumed that venous stenosis exists in PTC, there is controversy as to whether venous stenosis and elevated venous pressure are due to elevated ICP or constitute the proximal cause of PTC. ${ }^{14-16}$ The first explanation is an application of the Monroe-Kellie doctrine: Expansion of the brain within a fixed space will lead to the compression of venous sinuses, assuming that these are compressible structures, to vent CSF during systole when ICP increases. ${ }^{17}$ One cadaveric study suggested that the stenosis of the transverse sinus observed in IIH might be due to the presence of a large septum within the venous sinus. ${ }^{18}$

\section{Clinical Syndrome}

\section{Presentation}

PTC classically presents with headache and, frequently, vision changes in women with obesity of childbearing age. ${ }^{19}$ Head-

\section{Table 2: Criteria for diagnosing IIH}

1) If symptoms are present, they may only reflect those of generalized intracranial hypertension or papilledema

2) If signs are present, they may reflect only those of generalized intracranial hypertension or papilledema

3) Documented elevated ICP measured in the lateral decubitus position (findings of assessment of ICP by lumbar puncture are considered abnormal if above $20 \mathrm{~cm} \mathrm{H}_{2} \mathrm{O}$ in normal-weight individuals and $25 \mathrm{~mm}$ $\mathrm{H}_{2} \mathrm{O}$ in obese individuals ${ }^{20}$; $\mathrm{MRI}$ abnormal if above $20 \mathrm{~cm} \mathrm{H}_{2} \mathrm{O}$ in normalweight individuals and $25 \mathrm{~mm} \mathrm{H}_{2} \mathrm{O}$ in obese individuals ${ }^{20}$ )

4) Normal CSF composition

5) No evidence of hydrocephalus, mass, structural, or vascular lesion on MRI or contrast-enhanced CT for typical patients and on MRI and MR venography for all others

6) No other cause of intracranial hypertension identified

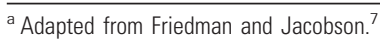

aches occur in nearly all (90\%-94\%) patients with PTC—-they are characteristically pressurelike, throbbing, and usually unremitting and occur with retro-ocular pain and may be accompanied by nausea. ${ }^{19,20}$ Vision loss is the most feared sequela of PTC, but most vision loss in this syndrome is transient in nature and occurs in approximately $68 \%-85 \%$ of patients. ${ }^{19-22}$ This vision loss less frequently takes the form of impairments in the visual field, with the typical impairment presenting as tunnel vision. ${ }^{19}$ Transient ischemia of the optic nerve due to pressure is thought to explain the transient vision changes. ${ }^{22}$ Pulse-synchronous tinnitus is another commonly reported symptom of PTC ( $58 \%$ of patients) and is often described as a unilateral "whooshing" sound by patients and may be exacerbated by positional changes and relieved by jugular compression. ${ }^{3,19,22}$ Other common symptoms include photopsia $(54 \%)$ and eye pain $(44 \%) .{ }^{22}$ More severe symptoms are less frequently encountered, yet diplopia $(38 \%)$ and vision loss $(30 \%)$ occur in a significant number of patients. ${ }^{22}$

Ophthalmologic signs of PTC consist of diminished visual acuity, visual field losses on formalized testing in nearly all patients, and, most strikingly, papilledema on funduscopic examination in $40 \%$ of patients. ${ }^{19,22}$ Visual loss is directly correlated with the extent of disc edema, which is thought to result from elevated IOP referred from elevated CSF pressure. ${ }^{23} \mathrm{An}$ atomic connections between the cranial fossa and orbit are thought to explain why ICP directly correlates with IOP. ${ }^{24}$ Absence of papilledema has been reported in many populations of patients with $\mathrm{IIH}$, but its absence may be more suggestive of an alternative etiology for headache and vision loss. ${ }^{19}$ Other funduscopic findings that may be seen in PTC are choroidal folds, parallel striae of alternating yellow crests, and darker troughs; choroidal folds compromise vision and can be seen with elevated ICP, even when papilledema has resolved. ${ }^{25,26}$ Cranial nerve palsies, usually of the abducens nerve (CN VI), may occur as well in as many as $10 \%-20 \%$ of patients. ${ }^{27}$ Rarely, facial nerve (CN VII) palsies may be associated with IIH; all of these $\mathrm{CN}$ palsies are thought to occur as a result of direct compression due to elevated ICP. ${ }^{22}$

\section{Epidemiology}

PTC is a rare condition, occurring in the general population with an incidence of 0.9 cases per 100,000 population in 2 epidemiologic studies in the United States. ${ }^{28,29}$ PTC demon- 


\begin{tabular}{lc}
\hline \multicolumn{2}{l}{ Table 3: Putative theories to explain elevated ICP in PTC } \\
\hline Proximal Etiology & \multicolumn{1}{c}{ Result } \\
\hline $\begin{array}{l}\text { Increased interstitial fluid (ISF) } \\
\text { volume }\end{array}$ & Increased cerebral volume \\
Increased blood volume & \\
$\begin{array}{l}\text { Increased tissue volume } \\
\text { Increased CSF production rate }\end{array}$ & Increased CSF volume \\
Increased CSF outflow resistance & Increased cerebral arterial pressure \\
Loss of cerebral autoregulation & Increased venous blood volume and \\
Increased cerebral venous pressure & increased ISF \\
& Reduced CSF outflow and increased \\
& CSF volume \\
\hline
\end{tabular}

${ }^{a}$ (Adapted from Walker. ${ }^{10}$ )

strates a strong female predominance, with a female/male ratio of $8: 1 .^{28}$

Reported risk factors for the development of PTC, including those discussed here, mostly originated from case reports, whereas very few controlled studies of PTC risk factors exist in the literature. ${ }^{20}$ As a result, some of these risk factors may be confounded by their coexistence in the at-risk population; for example, PCOS occurs more frequently in reproductive-age women with obesity, yet 1 study posited an association between PCOS and PTC while failing to control for the increased incidence of PCOS in women with obesity, who are already at increased risk of PTC. ${ }^{30}$

Classically, PTC is more commonly observed in overweight women of reproductive age. ${ }^{24}$ The incidence of PTC in women who are between 20 and 44 years of age and $20 \%$ above ideal body weight increases to 19.3 cases per 100,000 population; the odds ratio of PTC increases from 6.5 for a BMI of 25-29 to 26.0 for a BMI of $>35 .^{28,31}$ Obesity has long been associated with the development of PTC. ${ }^{10,31,32}$ One mechanistic theory proposes that obesity predisposes patients to having elevated intrabdominal pressure, elevated intrathoracic pressure, and thus elevated central venous pressure as the final common pathway leading to IIH. ${ }^{32}$ Randhawa and Van Stavern ${ }^{26}$ point out that this explanation fails to elucidate a female predominance. A recent study counters this theory of increased central venous pressure by discovering lower body (gynecoid) obesity as a risk factor for $\mathrm{IIH}$, which may suggest that increased estrogenicity, rather than elevated intrabdominal pressure, is the inciting element by which obesity propagates $\mathrm{IIH}^{33}$

In keeping with enhanced estrogenicity in the obese, others propose endocrinologic dysregulation leading to a prothrombotic state in obesity and, similarly, in pregnancy, as the pathway that promotes PTC. ${ }^{34}$ Other studies note increased leptin levels in obese patients with IIH compared with both obese and nonobese controls, ${ }^{35}$ while another group did not find significant differences between leptin levels of patients and controls. ${ }^{36}$ A recent study found similar CSF leptin levels in patients with PTC but noted significantly increased CSF levels of the inflammatory cytokine CCL2 ${ }^{37}$ Still another divergent explanation for obesity in PTC is that intracranial hypertension leads to hyperphagia; according to this theory, obesity is the result rather than the proximal cause of PTC. ${ }^{38}$ However, if PTC was the cause of obesity, one would expect more women to present with symptoms of increased ICP before weight gain under this theory. Thus, while obesity is long-established as a substantial risk factor for the development of PTC in women, it is uncertain whether the exact mechanism of this increased risk is related to a prothrombotic state, enhanced estrogenicity, inflammatory cytokine expression, endocrinologic dysfunction, or some other yet-to-be-ascertained mechanism.

While more common in reproductive-age women, PTC may occur as a slightly different clinical entity in other populations. ${ }^{4}$ These older patients are more likely to be men, present with different symptoms (fewer headaches, more visual changes), and have identifiable etiologies for elevated ICP, which arguably disqualify these patients from fitting the criteria for IIH on further clinical investigation. ${ }^{4}$ Men with PTC are less likely to be obese ( $25 \%$ versus $75 \%$ of women) and are at a twice greater risk of vision loss than women with PTC. ${ }^{39,40}$

PTC is rare within the pediatric population and generally occurs with greater frequency in postpubertal females, obesity becoming a significant risk factor with older age. ${ }^{41}$ PTC occurring in prepubertal children generally lacks any sex predilection or association with obesity and is thought to have less risk of permanent vision loss. ${ }^{42}$

\section{Management and Outcomes}

IIH and PTC were typically thought of as self-limiting conditions with little risk of major sequelae, which, in part, explains the historical name "benign intracranial hypertension"; however, severe deficits of visual acuity may occur in as many as one-quarter of patients without treatment. ${ }^{29}$ Recently, some racial disparities in outcome have been noted, in which black patients are more than 3 times as likely to have severe visual loss, even with equivalent access to treatment; severe visual loss was present in $23 \%$ of black patients versus $7 \%$ of nonblack patients. ${ }^{43}$ Patients with PTC also appear to have depression and anxiety to a greater extent than weight-matched controls and healthy controls. ${ }^{44}$ Health-related quality-of-life scores are also affected in PTC, with patients reporting significantly lower general health than controls and patients with other neuro-ophthalmologic conditions. ${ }^{31}$

Restoration of visual acuity and resolution of papilledema constitute the primary goals of management in PTC and the benchmark of relative success of a treatment technique. The fact that most cases of PTC spontaneously resolve and that most treatment studies lack control groups limits our interpretation of outcome measures reported for many treatment modalities. ${ }^{45}$ Conservative measures are typically recommended in patients without vision changes as the predominant presentation and consist of weight loss and medication. ${ }^{20}$ These conservative approaches may take time to take effect; as a result, frequent follow-up, including formal visual field testing at intervals of 1 month, 3 months, and then every 3-6 months, is advisable. ${ }^{20}$

Weight loss is thought to address 1 of the fundamental risk factors for recurrence of PTC and may reduce the risk of PTC and concomitant vision impairment through reductions of central venous pressure or a tentative endocrinologic mechanism. ${ }^{32}$ Gastric bypass surgery was associated with remittance of symptoms in all but 1 of 19 patients with a history PTC in 1 study; notably, symptoms only recurred in the 2 patients who regained weight following surgery. Another case report demonstrated a reduction of dural venous sinus pressure in $2 \mathrm{pa}-$ tients following bariatric surgery. ${ }^{46,47}$

Medications to treat and prevent recurrence of PTC typi- 


\begin{tabular}{|c|c|c|c|}
\hline & References & Sensitivity & Specificity \\
\hline \multirow[t]{2}{*}{ Empty sella } & Agid et al, $2006^{66}$ & $26.7 \%$ & $94.6 \%$ \\
\hline & Yuh et al, $2000^{64}$ & $2.5 \%$ & \\
\hline \multirow[t]{3}{*}{ Partially empty sella/decreased pituitary height } & Agid et al, $2006^{66}$ & $53.3 \%$ & $75 \%$ \\
\hline & Yuh et al, $2000^{64}$ & $80 \%$ & $92 \%$ \\
\hline & Brodsky and Vaphiades, $1998^{62}$ & $70 \%$ & \\
\hline \multirow[t]{3}{*}{ Flattened posterior globe/sclera } & Agid et al, $2006^{66}$ & $43.3 \%$ & $100 \%$ \\
\hline & Brodsky and Vaphiades, $1998^{62}$ & $80 \%$ & \\
\hline & Jinkins et al, $1996^{71}$ & $66.7 \%$ & \\
\hline \multirow[t]{2}{*}{ Enlarged ONS (perioptic subarachnoid space) } & Agid et al, $2006^{66}$ & $66.7 \%$ & $82.1 \%$ \\
\hline & Brodsky and Vaphiades, $1998^{62}$ & $45 \%$ & \\
\hline \multirow[t]{2}{*}{ Increased tortuosity of optic nerve } & Agid et al, $2006^{66}$ & $40 \%$ & $91.1 \%$ \\
\hline & Brodsky and Vaphiades, $1998^{62}$ & $40 \%$ & \\
\hline \multirow[t]{2}{*}{ Enhancement of optic nerve } & Agid et al, $2006^{66}$ & $6.7 \%$ & $98.2 \%$ \\
\hline & Brodsky and Vaphiades, $1998^{62}$ & $50 \%$ & \\
\hline \multirow[t]{2}{*}{ Intraocular protrusion of optic nerve head } & Agid et al, $2006^{66}$ & $3.3 \%$ & $100 \%$ \\
\hline & Brodsky and Vaphiades, $1998^{62}$ & $30 \%$ & \\
\hline Slitlike ventricles & Agid et al, $2006^{66}$ & $3.3 \%$ & $100 \%$ \\
\hline
\end{tabular}

cally have activity against carbonic anhydrase, which has been found to decrease the rate of CSF production, and thus address 1 of the proposed mechanisms of PTC. ${ }^{22}$ Acetazolamide is historically the most commonly used medication to treat PTC, but topiramate has been shown to have equal effect as a result of partial carbonic anhydrase-inhibition activity augmented by beneficial analgesic action against headaches and weight loss as a fortuitous side effect. ${ }^{15,26,48}$ Corticosteroids may result in temporary remission of PTC, but the risks of chronic steroid use in already obese patients preclude their use. Some clinicians advocate the use of corticosteroids in patients with severe headache, acute papilledema, and very high CSF pressure. $^{15,49}$

Therapeutic lumbar puncture is another common treatment method and addresses the issue of elevated CSF pressure directly by reducing it immediately; 1 older study suggests that the effects are short-lived, with a return to initial pressure within just $>1$ an hour. ${ }^{50}$ There is little published evidence comparing lumbar puncture with medication or other treatment modalities.

More aggressive measures for preventing sequelae of PTC are traditionally reserved for 2 groups of patients: those who continue to experience vision loss despite conservative management and those who initially present with rapid vision loss. Lumboperitoneal shunt surgery is a method in which the elevated CSF pressure is referred to the peritoneal cavity; this situation places the patient at risk of serious complications, ranging from shunt obstruction and shunt-related meningitis or abdominal infection to tonsillar herniation and death. ${ }^{20}$ Shunt failure occurs in roughly one-half of patients, and approximately one-tenth of patients have worsening vision following shunt surgery for IIH. As a result, shunt placement necessitates frequent neurologic follow-up. ${ }^{22,49}$

Many ophthalmologists advocate ONSF (also called decompression) as an alternative surgical approach associated with fewer complications and improved outcomes in the management of refractory PTC. ${ }^{20,51}$ In this approach, the surgeon cuts slits in the ONS behind the globe to allow CSF to leak from the subarachnoid space. ${ }^{22,49}$ Postoperative MR imaging showing fluid collections adjacent to the site of fenestration supports CSF extravasation into the orbit as the mechanism by which ONSF relieves symptoms. ${ }^{51}$ While some studies have alleged that the incidence of serious complications from ONSF is as low as $1 \%$, ONSF may result in worsening vision, infection, oculomotor dysfunction (albeit generally temporary), and death (in 1 older study). ${ }^{20,22,52}$ Following ONSF, visual acuity stabilized or improved in $94 \%$ of eyes and deteriorated in $6 \%$ of eyes in 2 studies. ${ }^{52,53}$ ONSF in the pediatric population improved visual acuity in two-thirds of patients, and vision worsened in $17 \%$ of patients. ${ }^{54}$

Some groups propose the use of venous sinus stent placement to treat IIH on the basis of several imaging studies showing stenosis of the cerebral venous sinuses and the presumption that venous outflow obstruction constitutes the underlying etiology of most cases of IIH. ${ }^{24,55}$ One group at the University of Cambridge reported success with 5 of 12 patients rendered asymptomatic and 4 of 8 patients demonstrating resolution of papilledema after venous sinus stent placement. ${ }^{55}$ Conversely, another group cited 3 cases of restenosis to counter the belief that stent placement is a universally applicable procedure in IIH; they argued that stenosis in IIH may be the result of the elevated ICP itself and not the cause of the increased ICP. ${ }^{56}$ Another researcher alleges that restenosis following stent placement is the result of failure to support all of the compliant portions of the venous sinus. ${ }^{57}$ Notably, the retrospective nonrandomized noncontrolled nature of these studies substantially limits objective assessment of the relative success of any of these treatment attempts.

\section{Imaging Findings}

Historically, imaging of patients with PTC served the purpose of excluding other pathology responsible for symptoms of increased ICP. ${ }^{58}$ Some of the earliest radiologic investigations of PTC demonstrated evidence of decreased ventricle size and sella turcica demineralization, thought to be a late manifestation of increased ICP, on plain film skull x-ray examination. ${ }^{59}$ The concept of decreased ventricle size as a sign of PTC emerged from these early studies. ${ }^{11,45}$ Early CT studies enabled clinicians to evaluate intracranial masses before lumbar puncture. ${ }^{58}$ Said and Rosman cautioned against using CT alone, which has the potential of missing important pathology that may explain increased ICP, especially in evaluating pediatric 


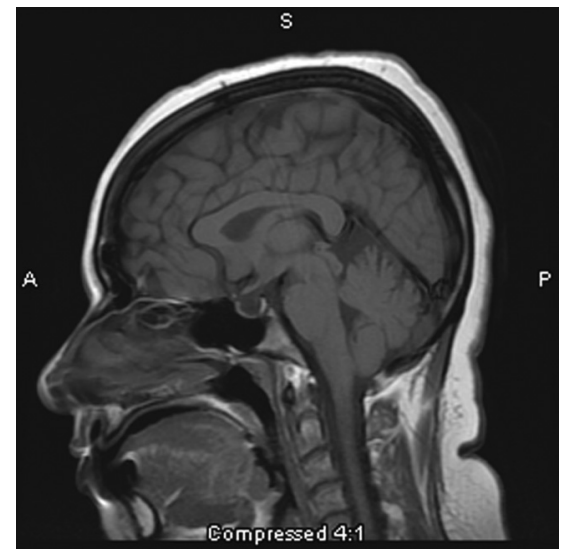

Fig 1. This 31-year-old woman presenting with headache is found to have an empty sella on sagittal T1-weighted MR imaging.

patients. ${ }^{60}$ The advent of MR imaging advanced the imaging paradigm in PTC from simply using imaging to rule out other processes (eg, space-occupying lesions) to detecting signs thought to indicate PTC itself.

\section{MR Imaging}

The American College of Radiologists, in their most recent appropriateness criteria, recommended the use of fat-suppressed contrast-enhanced MR imaging of the brain for the evaluation of any disorder involving vision changes. ${ }^{61}$ Several neuroimaging findings have been put forth as signs supporting intracranial hypertension or even more specifically PTC. Estimates of the occurrence, sensitivity, specificity, and relevance of these imaging signs vary widely in the published literature and clinical practice and are summarized in Table 4. These vast ranges of reported statistics and anecdotes most likely result from the varying effort in detecting particular signs at the expense of potentially ignoring other findings and technical differences (particularly in MR venography).

The "empty sella" sign (Fig 1) is associated with the longstanding effects of increased ICP and is thought to result from a downward herniation of an arachnocele through the diaphragma sella. ${ }^{45,62}$ It was 1 of the first signs noted on plain film $\mathrm{X}$-ray examination and subsequently on early MR imaging studies. ${ }^{59,63}$ The term "empty sella" should be reserved for studies in which the pituitary gland is not visible, and these cases tend to be later manifestations of increased ICP. ${ }^{64}$ There is a wide spectrum of pituitary height changes; thus, many cases of empty sella may, in fact, be better described as a partially empty sella or a compressed pituitary gland. The wide range of sensitivities and specificities reported reflects this ambiguity.

Transverse sinus narrowing (Fig 2) can be seen best on MR venography as well as on sagittal and axial MR imaging and is thought to represent the effect of increased ICP. ${ }^{15} \mathrm{~A}$ small or absent bony groove in the occiput in conjunction with the compressible nature of the transverse sinus makes this structure vulnerable to tapering with increased ICP. ${ }^{65}$ This particular imaging finding is more frequently noticed on MR venography studies, which are discussed at more length below. ${ }^{6}$

Posterior globe flattening is considered by some authors to be the sine qua non neuroimaging sign of PTC and can be seen on both CT and MR imaging but may be a more subtle finding

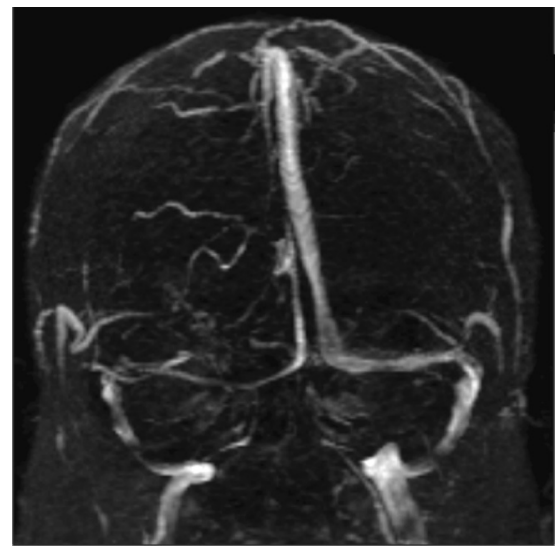

Fig 2. A narrowed right transverse sinus is noted in this 32-year-old woman, seen on MR venography, in addition to ONS enlargement and a partially empty sella on axial MR imaging.

subject to interpretation. ${ }^{66-68}$ Globe flattening may be explained by the direct correlation between elevated ICP and IOP via the transmission of elevated CSF pressure through the subarachnoid space, extending through the ONS to the posterior globe. ${ }^{24}$ One possible confounding explanation for posterior sclera flattening is its detection in the context of ocular hypotony; however, this condition is more rare, and this neuroimaging finding is more likely to be indicative of intracranial hypertension. ${ }^{68,69}$

Intraocular protrusion (Fig 3) is thought to occur in a manner similar to posterior globe flattening and is another sign associated with PTC. ${ }^{70,71}$ The optic nerve head is considered by some to be the most vulnerable site; thus, this finding on MR imaging may well correspond to the presence of visual symptoms, in light of its absence in patients with PTC lacking visual symptoms. ${ }^{70}$ This finding has also been reported in pediatric patients as well. ${ }^{72}$

ONS enlargement (Fig $4 A,-B$ ) appears as a widened ring of CSF around an optic nerve, which may appear compressed on coronal images, and as widened CSF signal intensity on either side of the optic nerve on axial images. ${ }^{62}$ Studies of the effectiveness of ONSF emphasize the importance of this imaging finding. ${ }^{73}$ ONS enlargement on T2-weighted MR imaging resolves in patients in whom ONSF is successful, while those patients who remain symptomatic still have enlarged ONSs. ${ }^{73}$

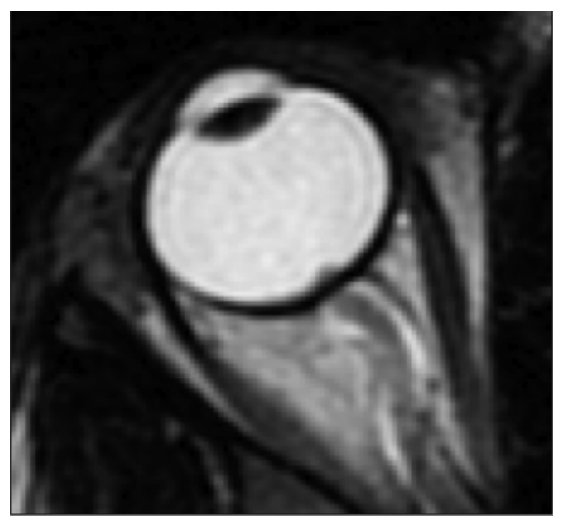

Fig 3. Protrusion of the right optic nerve head and horizontal tortuosity of the optic nerve are seen in this 21-year-old woman on axial T2-weighted MR imaging. Clinically, the patient presented with headaches, vision changes, and papilledema noted on examination. 

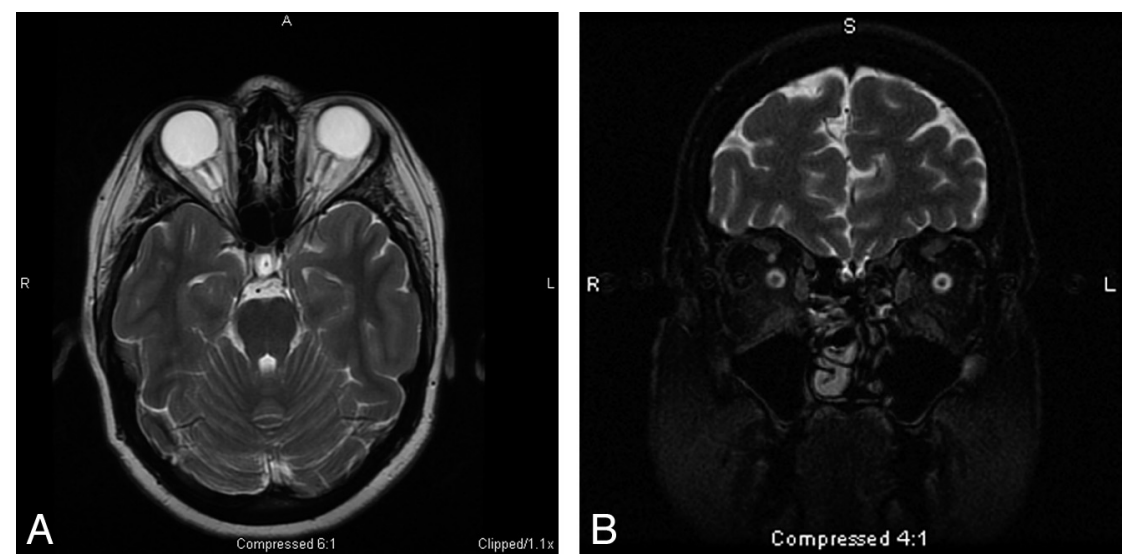

Fig 4. $A$, The ONS is widened with expanded CSF hyperintensity surrounding the optic nerve, seen on axial T2-weighted MR imaging in conjunction with posterior flattening of the globes. ONS widening is thought to coincide with papilledema, which is seen in this 27-year-old woman who presented with headaches. B, Coronal T2-weighted MR imaging in a 55-year-old woman with headache demonstrates increased peri-ONS space marked by hyperintense signal intensity surrounding the optic nerve.

Widening of the optic nerve has also been observed in childhood IIH. ${ }^{72}$ This association of ONS enlargement in PTC is supported by the converse association of the decreased diameter of the ONS in patients with CSF hypovolemia. ${ }^{74}$

Optic nerve tortuosity (Fig 3 ) has been associated with increased ICP; the distal and proximal points of fixation of the optic nerve enable it to kink freely in its course to the globe on protrusion of the intracranial contents under pressure. ${ }^{62}$ The sensitivity of observing optic nerve tortuosity in either the vertical or horizontal planes is dependent on section thickness. Horizontal tortuosity is thought to be less frequently visualized but more specific to intracranial hypertension.

Enhancement of the optic nerve is thought to be reflective of the same pathology leading to papilledema. Increased pressure referred from the cranial fossa generates venous congestion, capillary leakage, and possible breakdown of the bloodretinal barrier. ${ }^{75}$ To evaluate for this finding, one must use contrast. $^{62}$

Slitlike ventricles appear to be a poor neuroimaging sign of PTC in light of the their infrequent occurrence. ${ }^{66}$ This finding was first noted in older studies using ventriculography and also in early CT studies, but it appears to be an insignificant finding of little clinical use now. ${ }^{45,58,59}$

\section{MR Venography}

Previously, it was thought that MR venography should be reserved for use in atypical (eg, male, normal weight) patients to rule out sinovenous thrombosis. ${ }^{76}$ Now, many authors propose that any patient with suspected elevated intracranial hypertension undergo MR venography in addition to traditional MR orbital imaging to evaluate venous thrombosis or stenosis as the etiology of PTC symptoms. ${ }^{6,8,39,55}$ Newer imaging methods have enhanced detection of intracranial sinovenous stenoses previously undetected due to artifactual flow voids in the transverse sinuses on traditional time-of-flight MR venography. ${ }^{6}$ Higgins et al ${ }^{14}$ identified bilateral sinus flow gaps in $65 \%$ of patients with IIH by using 3D contrast-enhanced venography. By using a novel MR venography method, autotriggered elliptic centric-ordered imaging, Farb et $\mathrm{al}^{6}$ identi- fied venous stenoses in $90 \%$ of patients with IIH with a reported sensitivity and specificity of $93 \%$. These findings have similarly been replicated in the CT venography literature. ${ }^{77}$ Further advancements in MR venography may demonstrate stenosis of the venous sinuses to be an excellent indicator of the presence of elevated ICP. ${ }^{6}$

Yet another hypothesized imaging indicator of IIH seen on MR venography is increased total blood flow. ${ }^{78}$ In support of this finding, Bateman ${ }^{78}$ noted a $46 \%$ increase in total blood flow in 5 patients with IIH and decreased blood flow in 7 patients, with secondary intracranial hypertension due to thrombosis and arachnoid granulation in 1 patient. As with many research inquiries, this form of measurement is a relative calculation of blood flow in patients compared with controls and, as of yet, has no direct clinical application for evaluating patients with suspected PTC.

\section{Other Imaging Methods}

MR imaging measurement of intracranial elastance and its correlation with ICP is another technique that may prove fruitful in averting a more invasive measurement of ICP through lumbar puncture. ${ }^{79}$ Unfortunately, this imaging technique has only been studied in a limited population of healthy controls and those with chronically elevated ICP, as well as in baboons; moreover, complex calculations to correlate the elastance index with ICP preclude clinical use at present. ${ }^{79}$ If validated, this method would greatly improve the feasibility of repeated measurement of ICP as part of routine evaluation following treatment.

\section{Recommended Imaging Protocol}

In evaluating patients with headache, clinicians frequently order routine brain MR imaging studies; thus, the radiologist must be cognizant of possible indicators of PTC on axial T2weighted images as discussed in this article. If there is an additional clinical or imaging indication to suggest a greater likelihood of PTC, we recommend the addition of coronal T2weighted imaging of the orbits to assess ONS widening and MR venography to evaluate venous sinus thrombosis, sino- 
venous narrowing related to PTC, or congenital narrowing of the venous sinuses.

\section{Summary}

PTC is a poorly understood clinical entity with a variety of putative mechanisms, including excessive CSF production, impaired CSF resorption, and obstructed venous outflow. If PTC is clinically suspected, patients should be imaged by using an orbital MR imaging study to rule out secondary causes of intracranial hypertension as well as to assess signs frequently seen in PTC, including flattened posterior globes, increased ONS width, empty sella, increased tortuosity and enhancement of the optic nerve, and intraocular protrusion of the optic nerve head. Many, additionally, advocate MR venography to detect sinovenous stenosis, which has been seen in many patients with PTC. Whether sinovenous stenosis is the cause or the result of intracranial hypertension remains unresolved, and the issue of therapeutic stent placement in the venous sinuses is contentious. Much more in the way of randomized controlled clinical trials is needed in the study of this elusive condition.

\section{References}

1. Pearce JM. From pseudotumour cerebri to idiopathic intracranial hypertension. Pract Neurol 2009;9:353-56

2. Johnston I. The historical development of the pseudotumor concept. Neurosurg Focus 2001;11:1-9

3. Giuseffi V, Wall M, Siegel PZ, et al. Symptoms and disease associations in idiopathic intracranial hypertension (pseudotumor cerebri): a case-control study. Neurology 1991;41:239-44

4. Bandyopadhyay S, Jacobson DM. Clinical features of late-onset pseudotumor cerebri fulfilling the modified Dandy criteria. I Neuroophthalmol 2002;22:9-11

5. Sylaja PN, Ahsan Moosa NV, Radhakrishnan K, et al. Differential diagnosis of patients with intracranial sinus venous thrombosis-related isolated intracranial hypertension from those with idiopathic intracranial hypertension. J Neurol Sci 2003;215:9-12

6. Farb RI, Vanek I, Scott JN, et al. Idiopathic intracranial hypertension: the prevalence and morphology of sinovenous stenosis. Neurology 2003;60:1418-24

7. Friedman DI, Jacobson DM. Diagnostic criteria for idiopathic intracranial hypertension. Neurology 2002;59:1492-95

8. Szitkar B. A meningioma exclusively located inside the superior sagittal sinus responsible for intracranial hypertension. AJNR Am J Neuroradiol 2010;31:E57-58

9. Smith JL. Whence pseudotumor cerebri? J Clin Neuroophthalmol 1985;5:55-56

10. Walker RW. Idiopathic intracranial hypertension: any light on the mechanism of the pressure? J Neurol Neurosurg Psychiatry 2001;71:1-7

11. Levine DN. Ventricular size in pseudotumor cerebri and the theory of impaired CSF absorption. J Neurol Sci 2000;177:85-94

12. Najjar MW, Azzam NI, Khalifa MA. Pseudotumor cerebri: disordered cerebrospinal fluid hydrodynamics with extra-axial CSF collections. Pediatr Neurosurg 2005;41:212-15

13. Silberstein SD, McKinstry RC. The death of idiopathic intracranial hypertension? Neurology 2003;60:1406-07

14. Higgins JNP, Gillard JH, Owler BK, et al. MR venography in idiopathic intracranial hypertension: unappreciated and misunderstood. J Neurol Neurosurg Psychiatry 2004;75:621-25

15. Brazis PW. Pseudotumor cerebri. Curr Neurol Neurosci Rep 2004;4:111-16

16. Suzuki H, Takanashi J, Kobayashi K, et al. MR imaging of idiopathic intracranial hypertension. AJNR Am J Neuroradiol 2001;22:196-99

17. Bateman G. Stenoses in idiopathic intracranial hypertension: to stent or not to stent? AJNR Am J Neuroradiol 2008;29:215

18. Subramaniam RM, Tress BM, King JO, et al. Transverse sinus septum: a new etiology of idiopathic intracranial hypertension? Australas Radiol 2004;48:114-16

19. Friedman DI. Pseudotumor cerebri. In: Levin LA, Arnold AC, eds. NeuroOphthalmology: The Practical Guide. New York: Thieme; 2005:183-86

20. Lueck CJ, McIlwaine GG. Idiopathic intracranial hypertension. Pract Neurol 2002:262-71

21. Rowe FJ, Sarkies NJ. Assessment of visual function in idiopathic intracranial hypertension: a prospective study. Eye 1994;12:111-18
22. Wall M. Idiopathic intracranial hypertension. Neurol Clin 2010;28:593-617

23. Wall $M$. The morphology of visual field damage in idiopathic intracranial hypertension: an anatomic region analysis. In: Mills RP, Heijl A, eds. Perimetry Update 1990/1991. Amsterdam, the Netherlands: Kugler Publishers; 1991:20-27

24. Sajjadi SA, Harirchian MH, Sheikhbahaei N, et al. The relation between intracranial and intraocular pressures: study of $\mathbf{5 0}$ patients. Ann Neurol 2006;59:867-70

25. Griebel SR, Kosmorsky GS. Choroidal folds associated with increased intracranial pressure. Am J Ophthalmol 2000;129:513-16

26. Randhawa S, Van Stavern GP. Idiopathic intracranial hypertension (pseudotumor cerebri). Curr Opin Ophthalmol 2008;19:445-53

27. Wall $\mathrm{M}$, George D. Idiopathic intracranial hypertension: a prospective study of 50 patients. Brain 1991;114:155-80

28. Durcan FJ, Corbett JJ, Wall M. The incidence of pseudotumor cerebri: population studies in Iowa and Louisiana. Arch Neurol 1988;45:875-77

29. Radhakrishnan K, Ahlskog JE, Cross SA, et al. Idiopathic intracranial hypertension (pseudotumor cerebri): descriptive epidemiology in Rochester, Minn, 1976 to 1990. Arch Neurol 1993;50:78-80

30. Glueck CJ, Iyengar S, Goldenberg $\mathrm{N}$, et al. Idiopathic intracrania hypertension: associations with coagulation disorders and polycystic-ovary syndrome. J Lab Clin Med 2003;142:35-45

31. Daniels AB, Liu GT, Volpe NJ, et al. Profiles of obesity, weight gain, and quality of life in idiopathic intracranial hypertension (pseudotumor cerebri). Am J Ophthalmol 2007;143:635-41

32. Sugerman HJ, DeMaria EJ, Felton WL, et al. Increased intra-abdominal pressure and cardiac filling pressures in obesity-associated pseudotumor cerebri. Neurology 1997;49:507-11

33. Kesler A, Kliper E, Shenkerman G, et al. Idiopathic intracranial hypertension is associated with lower body adiposity. Ophthalmology 2010;117:169-74

34. Ooi LY, Walker BR, Bodkin PA, et al. Idiopathic intracranial hypertension: can studies of obesity provide the key to understanding pathogenesis? Br J Neurosurg 2008;22:187-94

35. Lampl Y, Eshel Y, Kessler A, et al. Serum leptin level in women with idiopathic intracranial hypertension. J Neurol Neurosurg Psychiatry 2002;72:642-43

36. Subramanian PS, Goldenberg-Cohen N, Shukla S, et al. Plasma ghrelin levels are normal in obese patients with idiopathic intracranial hypertension (pseudotumor cerebri). Am J Ophthalmol 2004;138:109-13

37. Dhungana S, Sharrack B, Woodroofe N. Cytokines and chemokines in idiopathic intracranial hypertension. Headache 2009;49:282-85

38. Hannerz J, Ericson K. The relationship between idiopathic intracranial hypertension and obesity. Headache 2009;49:178-84

39. Kesler A, Goldhammer Y, Gadoth N. Do men with pseudotumor cerebri share the same characteristics as women? A retrospective review of 141 cases. $\mathrm{J} \mathrm{Neu}$ roophthalmol 2001;21:15-17

40. Bruce BB, Kedar S, Van Stavern GP, et al. Idiopathic intracranial hypertension in men. Neurology 2009;27:304-09

41. Balcer LJ, Liu GT, Forman S, et al. Idiopathic intracranial hypertension: relation of age and obesity in children. Neurology 1999;52:870-72

42. Kesler A, Fattal-Valevski A. Idiopathic intracranial hypertension in the pediatric population. J Child Neurol 2002;17:745-48

43. Bruce BB, Preechawat $\mathrm{P}$, Newman NJ, et al. Racial differences in idiopathic intracranial hypertension. Neurology 2008;70:861-67

44. Kleinschmidt JJ, Digre KB, Hanover R. Idiopathic intracranial hypertension: relationship to depression, anxiety, and quality of life. Neurology 2000;54:319-24

45. George AE. Idiopathic intracranial hypertension: pathogenesis and the role of MR imaging. Radiology 1989;170:21-22

46. Sugerman HJ, Felton WL, Sismanis A, et al. Gastric surgery for pseudotumor cerebri associated with severe obesity. Ann Surg 1999;229:634-40

47. Nadkarni T, Rekate HL, Wallace D. Resolution of pseudotumor cerebri after bariatric surgery for related obesity: case report. J Neurosurg 2004;101:878-80

48. Celebisoy N, Gokcay F, Sirin H, et al. Treatment of idiopathic intracranial hypertension: topiramate vs acetazolamide, an open-label study. Acta Neurol Scand 2007;116:322-27

49. Matthews MK, Sergott RC, Savino PJ. Pseudotumor cerebri. Curr Opin Ophthalmol 2003; 14:364-70

50. Johnston I, Paterson A. Benign intracranial hypertension. II. CSF pressure and circulation. Brain 1974;97:301-12

51. Yazici Z, Yazici B, Tuncel E. Findings of magnetic resonance imaging after optic nerve sheath decompression in patients with idiopathic intracranial hypertension. Am J Ophthalmol 2007;144:429-35

52. Banta JT, Farris BK. Pseudotumor cerebri and optic nerve sheath decompression. Ophthalmology 2000;107:1907-12

53. Nithyanandam S, Manayath GJ, Battu RR. Optic nerve sheath decompression for visual loss in intracranial hypertension: report from a tertiary care center in South India. Indian J Ophthalmol 2008;56:115-20

54. Lee AG, Patrinely JR, Edmond JC. Optic nerve sheath decompression in pediatric pseudotumor cerebri. Ophthalmic Surg Lasers 1998;29:514-17

55. Higgins JNP, Cousins C, Owler BK, et al. Idiopathic intracranial hypertension: 12 cases treated by venous sinus stenting. J Neurol Neurosurg Psychiatry 2003;74:1662-66 
56. Rohr A, Dorner L, Stingele R, et al. Reversibility of venous sinus obstruction in idiopathic intracranial hypertension. AJNR Am J Neuroradiol 2007;28:656-59

57. Bateman G. Stenoses in idiopathic intracranial hypertension: to stent or not to stent? AJNR Am J Neuroradiol 2008;29:215

58. Lightfoote WE, Pressman BD. Increased intracranial pressure: evaluation by computerized tomography. Am J Roentgenol Radium Ther Nucl Med 1975;124:195-98

59. Jacobson HG, Shapiro JH. Pseudotumor cerebri. Radiology 1964;82:202-10

60. Said RR, Rosman NP. A negative cranial computed tomographic scan is not adequate to support a diagnosis of pseudotumor cerebri. J Child Neurol 2004;19:609-13

61. Wippold FJ. Orbits, vision, and visual loss. AJNR Am J Neuroradiol 2010;31:196-98

62. Brodsky MC, Vaphiades M. Magnetic resonance imaging in pseudotumor cerebri. Ophthalmology 1998;105:1686-93

63. Silbergleit R, Junck L, Gebarski SS, et al. Idiopathic intracranial hypertension (pseudotumor cerebri): MR imaging. Radiology 1989;170:207-09

64. Yuh WT, Zhu M, Taoka T, et al. MR imaging of pituitary morphology in idiopathic intracranial hypertension. J Magn Reson Imaging 2000;12:808-13

65. Connor SE, Siddiqui MA, Stewart VR, et al. The relationship of transverse sinus stenosis to bony groove dimensions provides an insight into the aetiology of idiopathic intracranial hypertension. Neuroradiology 2008;50:9991004. Epub 2008 Jul 12

66. Agid R, Farb RI, Willinsky RA, et al. Idiopathic intracranial hypertension: the validity of cross-sectional neuroimaging signs. Neuroradiology 2006;48:521-27

67. Madill SA, Connor SE. Computed tomography demonstrates short axial globe length in cases with idiopathic intracranial hypertension. J Neuroophthalmol $2005 ; 25: 180-84$
68. Brodsky MC. Flattening of the posterior sclera: hypotony or elevated intracranial pressure? Am J Ophthalmol 2004;138:511

69. Westfall AC, Ng JD, Samples JR, et al. Hypotonus maculopathy: magnetic resonance appearance. Am J Ophthalmol 2004;137:563-66

70. Gass A, Barker GJ, Riordan-Eva P, et al. MRI of the optic nerve in benign intracranial hypertension. Neuroradiology 1996;38:769-73

71. Jinkins JR, Athale $S$, Xiong L, et al. MR of optic papilla protrusion in patients with high intracranial pressure. AJNR Am J Neuroradiol 1996;17:665-68

72. Mandelstam S, Moon A. MRI of optic disc edema in childhood idiopathic intracranial hypertension. Pediatr Radiol 2004;34:362

73. Sallomi D, Hibbert TJ, Sanders MD, et al. The MRI appearance of the optic nerve sheath following fenestration for benign intracranial hypertension. Eur Radiol 1998;8:1193-96

74. Watanabe A, Horikoshi T, Uchida M, et al. Decreased diameter of the optic nerve sheath associated with CSF hypovolemia. AJNR Am J Neuroradio 2008;29:863-64

75. Manfre L, Lagalla R, Mangiameli A, et al. Idiopathic intracranial hypertension: orbital MRI. Neuroradiology 1995;37:459-61

76. Lee AG, Brazis PW. Magnetic resonance venography in idiopathic pseudotumor cerebri. J Neuroophthalmol 2000;20:12-13

77. Higgins JN, Tipper G, Varley M, et al. Transverse sinus stenoses in benign intracranial hypertension demonstrated on CT venography. Br J Neurosurg 2005; 19:137-40

78. Bateman GA. Vascular hydraulics associated with idiopathic and secondary intracranial hypertension. AJNR Am J Neuroradiol 2002;23:1180-86

79. Alperin NJ, Lee SH, Loth F, et al. MR-intracranial pressure (ICP): a method to measure intracranial elastance and pressure noninvasively by means of MR imaging: baboon and human study. Radiology 2000;217:877-85 